\title{
Circulating cytokeratin-18 and tumour necrosis factor- $a$ in patients with alcoholic liver cirrhosis
}

Andrzej Prystupa', Paweł Kiciński², Jarosław Niedziałek ${ }^{3}$, Anna Toruń-Jurkowska4, Anna Boguszewska-Czubara ${ }^{5}$, Dorota Luchowska-Kocot ${ }^{5}$, Andrzej Ochal ${ }^{6}$, Mariusz Jojczuk ${ }^{6}$, Jarosław Sak ${ }^{7}$

${ }^{1}$ Department of Internal Diseases, Medical University of Lublin, Poland

2 Department of Family Medicine, Medical University of Lublin, Poland

${ }^{3}$ Individual Medical Practice, Lublin, Poland

${ }^{4}$ Department of Mathematics and Medical Biostatistics, Medical University of Lublin, Poland

${ }^{5}$ Department of Medical Chemistry, Medical University of Lublin, Poland

${ }^{6}$ Department of Trauma Surgery and Emergency Medicine, Medical University of Lublin, Poland

7 Department of Ethics and Human Philosophy, Medical University of Lublin, Poland

Prystupa A, Kicński P, Niedziałek J, Toruń-Jurkowska A, Boguszewska-Czubara A, Luchowska-Kocot D, Ochal A, Jojczuk M, Sak J. Circulating cytokeratin-18 and tumour necrosis factor-a in patients with alcoholic liver cirrhosis. J Pre-Clin Clin Res. 2016 ; 10(2): 87-90. doi: $10.5604 / 18982395.1227562$

\section{Abstract}

Aims. The aim of the study was to assess the usefulness of TNF- $a$ and CK-18 as diagnostic markers of alcoholic liver cirrhosis. Additionally, the effects of the stage of liver cirrhosis on concentrations of TNF- $a$ and CK-18, as well as their correlation, were evaluated.

Materials and method. Sixty-two patients with alcoholic liver cirrhosis treated in various hospitals were randomly enrolled. The stage of cirrhosis was assessed according to the Child-Turcotte-Pugh scoring system. The control group consisted of 31 healthy people without liver disease. Concentrations of TNF- $a$ and cytokeratin-18 in blood plasma of patients and controls were measured using the sandwich enzyme immunoassay technique, with commercially available quantitative ELISA test kits.

Results. The concentration of CK-18 was statistically higher in patients with alcoholic liver cirrhosis, compared to the control group. The concentration of TNF-a was significantly higher in patients with alcoholic liver cirrhosis, compared to the control group. Higher concentrations of TNF-a were found only in patients with stage C and B alcoholic liver cirrhosis, compared to healthy persons.

Conclusions. The levels of TNF- $a$ and total CK-18 were higher in patients with alcoholic liver cirrhosis than in healthy individuals. No correlation was found between the level of CK-18 and stage of liver cirrhosis.

\section{Key words}

liver cirrhosis, alcohol, apoptosis markers

\section{INTRODUCTION}

Liver cirrhosis is the final stage of many liver diseases. In Europe, the majority of liver cirrhosis cases are associated with alcoholic liver cirrhosis and chronic viral hepatitis [1]. Cirrhosis is defined as the development of regenerative nodules surrounded by fibrous bands in response to chronic liver injury that leads to portal hypertension and end-stage liver disease [2]. Fibrosis reflects a loss of homeostasis between fibrogenesis and matrix degeneration. The first step of fibrosis is the activation of stellate cells by tumour necrosis factor- $\alpha$ (TNF- $\alpha$ ) released from the injured hepatocytes [3]. TNF- $\alpha$ is considered to be the most potent inducer of apoptosis [4]. Hepatocyte apoptosis has been recognized as a mechanism of liver injury that may contribute to fibrogenesis. Apoptosis of hepatocytes is a significant histological feature of alcoholic liver diseases. Serum M30-M65 levels can provide information about necrosis and apoptotic activity. M65 assay expresses all fragments of cytokeratin-18 (CK-18) released into the circulation during both cell necrosis and apoptosis [5]. On the contrary, M30 assay assesses the level of caspase-cleaved CK-18, but not of native, uncleaved CK-

Address for correspondence: Paweł Kiciński, Department of Family Medicine, Medical University, Staszica 11, 20-081 Lublin, Poland

E-mail: pawelkici@wp.pl

Received: 12 August 2016; accepted: 26 August 2016
18 , and therefore represents a specific quantitative test for apoptosis in epithelial cells [6].

The PubMed database does not include any studies regarding the use of a M65 biomarker (CK-18) for the diagnosis of alcoholic liver cirrhosis, assessment of its stage and correlation with the level of TNF- $\alpha$.

\section{OBJECTIVE}

The aim of the study was to assess the usefulness of TNFa and CK-18 as diagnostic markers of alcoholic liver cirrhosis. Dditionally, the effects of the stage of liver cirrhosis on concentrations of TNF- $\alpha$ and CK-18 and their correlation were evaluated.

\section{MATERIALS AND METHOD}

Patients with alcoholic liver cirrhosis treated in various hospitals of the Lublin region of eastern Poland were randomly enrolled. The study group included 46 male and 16 female patients. All patients presented a history of heavy alcohol consumption in the absence of positivity for serological viral markers. The diagnosis of liver cirrhosis was based on clinical features, laboratory tests, abdominal ultrasound and history 
of heavy alcohol consumption. Patients with concomitant presence of alcoholic hepatitis were excluded. The stages of cirrhosis were assessed according to the Child-Turcotte-Pugh criteria (Child-Pugh score) as P-Ch A, P-Ch B, P-Ch C. The control group consisted of 31 (25 male and 6 female) healthy individuals without liver disease, who did not drink alcohol. Both clinical assessment and laboratory tests were used to exclude underlying liver diseases in the control group. There were no significant differences between age and gender distribution in the study and control groups. Characteristics of the study population are given in Tables 1 and 2 .

Table 1. Characteristics of patients with alcoholic liver cirrhosis ( $\mathrm{P}-\mathrm{Ch}$ stage $A, B, C$ ) and healthy controls (C)

\begin{tabular}{|c|c|c|c|c|}
\hline & $\begin{array}{c}\mathbf{C} \\
(n=31)\end{array}$ & $\begin{array}{l}\text { P-Ch A } \\
(n=17)\end{array}$ & $\begin{array}{l}\text { P-Ch B } \\
(n=21)\end{array}$ & $\begin{array}{l}\text { P-Ch C } \\
(n=24)\end{array}$ \\
\hline Age (years) & $56.51 \pm 8.81$ & $52.50 \pm 16.11$ & $54.00 \pm 12.19$ & $50.71 \pm 10.00$ \\
\hline $\begin{array}{l}\text { Gender } \\
\text { (male/female) (n) }\end{array}$ & $25 / 6$ & $14 / 4$ & $13 / 8$ & $20 / 4$ \\
\hline Body weight (kg) & $75.63 \pm 9.90$ & $66.33 \pm 11.93$ & $84.84 \pm 27.11$ & $85.91 \pm 21.76$ \\
\hline Height $(\mathrm{cm})$ & $175.54 \pm 10.31$ & $171.33 \pm 9.86$ & $177.36 \pm 11.40$ & $175.45 \pm 6.69$ \\
\hline $\begin{array}{l}\text { Drinking period } \\
\text { (years) }\end{array}$ & Occasionally & $11.16 \pm 7.40$ & $13.86 \pm 7.06$ & $18.17 \pm 10.73$ \\
\hline \multicolumn{5}{|l|}{$\begin{array}{l}\text { Existing medical } \\
\text { symptoms }\end{array}$} \\
\hline Ascites (n) & 0 & 1 & 14 & 15 \\
\hline $\begin{array}{l}\text { Encephalophaty } \\
\text { (n) }\end{array}$ & 0 & 3 & 8 & 18 \\
\hline $\begin{array}{l}\text { Oesophageal } \\
\text { varices }(n)\end{array}$ & 0 & 2 & 9 & 16 \\
\hline
\end{tabular}

Table 2. Biochemical data of the study participants with alcoholic liver cirrhosis (P-Ch stage A, B, C) and healthy controls (C)

\begin{tabular}{|c|c|c|c|c|}
\hline & $\begin{array}{c}\mathbf{C} \\
(n=31)\end{array}$ & $\begin{array}{l}\text { P-Ch A } \\
(n=17)\end{array}$ & $\begin{array}{l}\text { P-Ch B } \\
(n=21)\end{array}$ & $\begin{array}{l}\text { P-Ch C } \\
(n=24)\end{array}$ \\
\hline ilirubin (mg/dl) & $0.65 \pm 0.22$ & $2.70 \pm 0.95$ & $12.31 \pm 4.48$ & $15.75 \pm 4.87$ \\
\hline lbumin (g/dl) & $5.23 \pm 0.54$ & $4.00 \pm 0.67$ & $3.80 \pm 0.84$ & $2.42 \pm 0.48$ \\
\hline $\mathrm{LT}(\mathrm{U} / \mathrm{I})$ & $20.24 \pm 8.55$ & $99.00 \pm 221.00$ & $41,57 \pm 29.48$ & $61.24 \pm 104.60$ \\
\hline ST (IU/I) & $17.82 \pm 5.04$ & $143.00 \pm 249.0$ & $96.67 \pm 101.57$ & $132 \pm 202,06$ \\
\hline GGTP (IU/I) & $20.41 \pm 8.98$ & $313.75 \pm 27.96$ & $642.24 \pm 70.04$ & $749.48 \pm 72.55$ \\
\hline Urea & $24.40 \pm 10.08$ & 3 & 4 & .39 \\
\hline $\begin{array}{l}\text { Blood platelets } \\
(\mathrm{K} / \mu \mathrm{l})\end{array}$ & $340.2 \pm 7.97$ & $166.75 \pm 11.96$ & $135.46 \pm 12.28$ & $105.33 \pm 7.02$ \\
\hline INR & $1.27 \pm 0.16$ & $1.30 \pm 0.21$ & $1.39 \pm 0.23$ & $2.01 \pm 0.90$ \\
\hline MCV (fl) & $86.00 \pm 7.25$ & $95.97 \pm 9.36$ & $97.09 \pm 6.27$ & $103.07 \pm 6.09$ \\
\hline $\begin{array}{l}\text { C-reactive } \\
\text { protein }(\mathrm{mg} / \mathrm{L})\end{array}$ & $2.51 \pm 2.29$ & $22.32 \pm 19.89$ & $18.63 \pm 17.94$ & $13.87 \pm 12.81$ \\
\hline $\mathrm{Va}(\mathrm{mmol} / \mathrm{l})$ & $139.50 \pm 3.45$ & $129.75 \pm 10.50$ & $134.05 \pm 4.78$ & $131.85 \pm 8.41$ \\
\hline $\mathrm{K}(\mathrm{mmol} / \mathrm{l})$ & $4.18 \pm 0.33$ & $3.59 \pm 0.42$ & $4.07 \pm 0.77$ & $3.86 \pm 0.60$ \\
\hline
\end{tabular}

Normal range: bilirubin (0-1.2 mg/dl), albumin (3.5-5.20 g/dl); ALT - alanine aminotransferase (5-40 U/I); AST - aspartate aminotransferase (5-40 IU/I); GGTP- Gamma-glutamyl transpeptidase (11-50 IU/I): urea (21-43 mg/dl): blood platelets (120-400 K/uL): INR (0.86-1.30): MCV (80-94 fl); C-reactive protein ( $<5 \mathrm{mg} / \mathrm{L})$; K - potassium (3.5-5.1 mmol/l); $\mathrm{Na}$ - sodium (136-145 mmol/l).

The study protocol was approved by the Ethic Committee of Medical University of Lublin. All subjects gave their written consent.

The material for the study was peripheral blood obtained from the ulnar vein. After an 8-12-hour overnight fast between 20:00-10.00, blood samples were collected into clot tubes in the volume of $7 \mathrm{ml}$. Serum was separated by centrifugation for $10 \mathrm{~min}$ at $1,000 \mathrm{rpm}$, aliquoted and stored frozen at $-20^{\circ} \mathrm{C}$ until analysis.
The serum total M65 EpiDeath concentration was determined using an M65 EpiDeath ${ }^{\circledR}$ ELISA (Vivalavida $\mathrm{AB})$ kit according to the manufacturer's instructions. The M65 EpiDeath ${ }^{\circledast}$ ELISA is a solid-phase sandwich enzyme immunoassay that can be used to assess the death of epithelial cells by measuring the release of cytokeratin-18 (CK-18) protein. The CK-18 levels reflect the amount of total epithelial cell death, regardless of the cause of death, due to apoptosis or necrosis. The minimum detectable concentration of CK-18 in the M65 EpiDeath ${ }^{\circledast}$ ELISA is $25 \mathrm{U} / \mathrm{L}$.

The serum TNF- $a$ concentration was determined applying the ELISA kit for Tumor Necrosis Factor Alpha (Cloud-Clone Corp.) according to the manufacturer's protocol. The kit is a sandwich enzyme immunoassay for in vitro quantitative measurement of TNF- $\alpha$ in human serum. The minimum detectable dose of TNF- $\alpha$ is typically $<0.54 \mathrm{pg} / \mathrm{mL}$.

Statistical analysis. The collected material was statistically analysed using STATISTICA 10 PL. Continuous variables were expressed as mean \pm standard deviation (SD). Before calculations, variables were checked for normality using the Shapiro-Wilk test; the Brown-Forythe's test was applied to test equality of variances. To compare continuous variables between two groups (in the control and the study group), the t-Student test was used when normally distributed, and Mann-Whitney test when non-normally distributed. To compare the results between dependent variables, CK-18 and TNF- $\alpha$ and the independent variable 'group', which divide the population into four categories: controls and cirrhotic patients with Child-Pugh classes A, B, and C, the KruskalWallis rank test was used, a nonparametric equivalent of ANOVA. The Dunn test was applied for detailed identification of statistically different groups. Correlations among variables were with the Spearmans' rank correlation test. For all tests, $\mathrm{p}<0.05$ was considered as statistically significant.

\section{RESULTS}

The concentration of CK-18 was significantly higher in patients with alcoholic liver cirrhosis $(407.3 \pm 198.21 \mathrm{U} / \mathrm{L})$, compared to the control group $(201.67 \pm 43.63 \mathrm{U} / \mathrm{L})(\mathrm{p}<0.0001$, Mann-Whitney test).

The concentration of TNF- $\alpha$ was found to be significantly higher in the group with alcoholic liver cirrhosis $(3.42 \pm 2.75 \mathrm{pg} / \mathrm{mL})$, as compared to the control group $(1,645 \pm 0.945 \mathrm{pg} / \mathrm{mL})(\mathrm{p}<0.001$, Mann-Whitney test $)$.

Moreover, with the Kruskal-Wallis test we analyzed the effects of the liver cirrhosis stages according to the ChildTurcotte-Pugh (P-Ch) criteria on concentrations of CK-18 and TNF- $\alpha$ (Figs. 1, 2; Tab. 3). The highest level of CK-18 was observed in patients with stage C liver cirrhosis $(429.39 \pm 351.2$ $\mathrm{U} / \mathrm{L})$. Detailed analysis enabled the demonstration of significantly lower CK-18 concentrations in the control group than those in patients with stage $C$ liver cirrhosis $(p<0.001)$. Moreover, significantly lower concentrations of CK-18 were found in the control group than in patients with stage A and B liver cirrhosis (both $\mathrm{p}<0.001$ ). Interestingly, differences in CK-18 concentrations among the individual stages of alcoholic liver cirrhosis (P-Ch A, B and C) were not statistically significant (Fig. 1).

Differences in TNF- $\alpha$ concentrations among the subgroups analysed were found to be statistically significant. Analysis 
Table 3. Concentrations of CK-18 and TNF- $a$ in patients with various stages of alcoholic liver cirrhosis (P-Ch A, B, C) and in controls

\begin{tabular}{lcc}
\hline Group & CK-18 (U/L) & TNF-a $(\mathbf{p g} / \mathbf{m L})$ \\
\hline Control $(n=31)$ & $201.67 \pm 43.63^{*}$ & $1.645 \pm 0.945^{* *}$ \\
\hline P-Ch A $(n=17)$ & $415.02 \pm 195.5$ & $2.161 \pm 1.365^{* *}$ \\
\hline P-Ch B $(n=21)$ & $375.82 \pm 179.9^{*}$ & $3.301 \pm 1.738^{* *}$ \\
\hline P-Ch C $(n=24)$ & $429.39 \pm 351.2^{*}$ & $4.415 \pm 3.721^{* *}$ \\
\hline All cirrhotic patients $(n=62)$ & $407.3 \pm 198.21$ & $3.42 \pm 2.75$ \\
\hline
\end{tabular}

* $\mathrm{p}<0.001$ for control group vs $\mathrm{P}$-Ch $\mathrm{A}$ and control group vs $\mathrm{P}$-Ch $C$

${ }^{* *} \mathrm{p}<0.001$ for control group vs $\mathrm{P}-\mathrm{Ch} \mathrm{A}$; control group vs $\mathrm{P}-\mathrm{Ch} \mathrm{B}$ and control group vs $\mathrm{P}-\mathrm{Ch} \mathrm{C}$

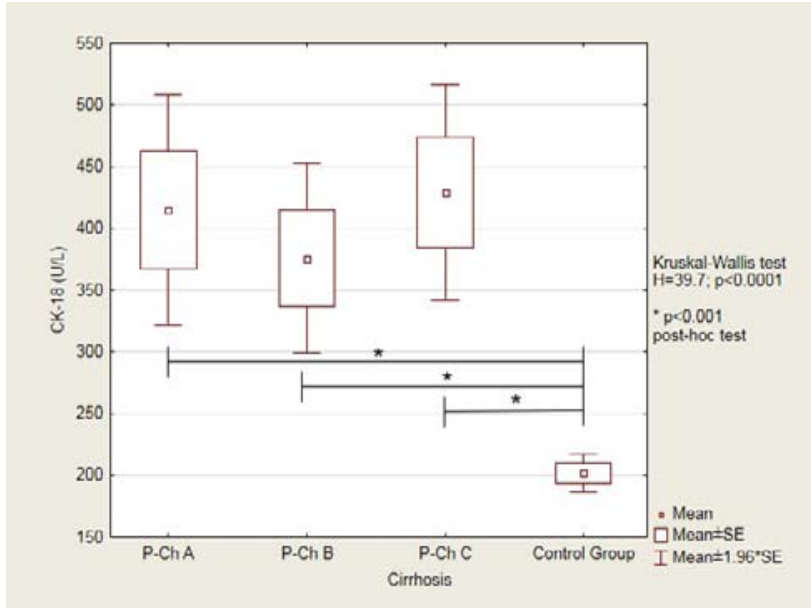

Figure 1. Concentrations of $C K-18$ in patients with Child-Pugh stage $A, B$ and $C$ alcoholic liver cirrhosis, and in the control group (C)

revealed significantly higher TNF-a concentrations in the group of patients with stage $\mathrm{C}$ alcoholic liver cirrhosis $(4.415 \pm 3.721 \mathrm{pg} / \mathrm{mL})$, compared to the control group $(1,645 \pm 0.945 \mathrm{pg} / \mathrm{mL} ; \mathrm{p}<0.001)$. A significantly higher concentration of TNF- $\alpha$ was found in stage B liver cirrhosis $(3.301 \pm 1.738 \mathrm{pg} / \mathrm{mL})$ than in the control group $(\mathrm{p}<0.001)$ (Fig. 2).

No correlation was observed between the concentration of TNF- $\alpha$ and of CK-18 in the group of patients with alcoholic liver cirrhosis ( $\mathrm{r}=0.15, \mathrm{p}>0.05)$. Furthermore, no correlations were observed between TNF- $\alpha$ and CK-18 levels versus albumin, total bilirubin and INR levels ( $\mathrm{p}>0.05)$.

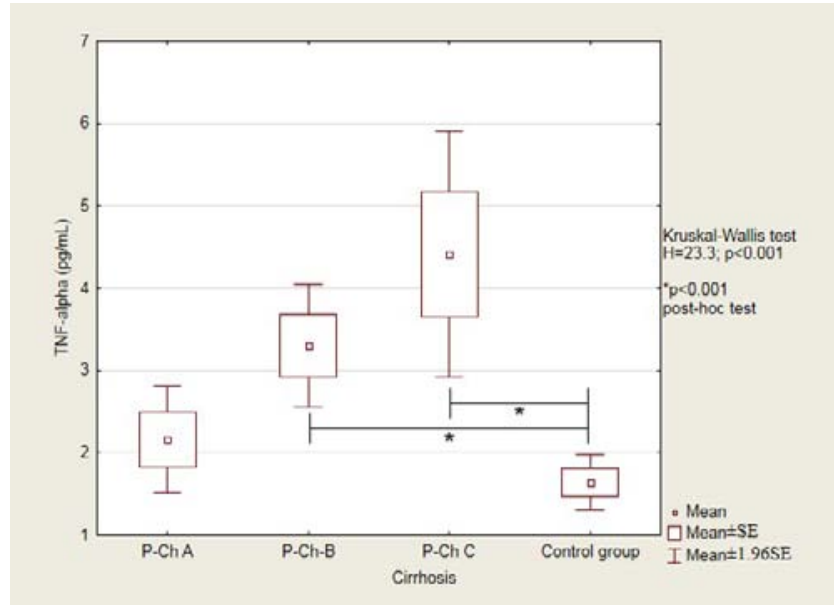

Figure 2. Concentrations of TNF- $\alpha$ in patients with Child-Pugh stage $A, B$ and $C$ alcoholic liver cirrhosis, and in the control group (C)

\section{DISCUSSION}

These findings demonstrated statistically significantly higher concentrations of CK-18 and TNF- $\alpha$ in the group of patients with alcoholic liver cirrhosis.

Tumour necrosis factor $\alpha$, a pro-inflammatory cytokine produced by activated Kupffer cells, has been implicated as a mediator of hepatocyte death involved in the pathophysiology of various conditions, including viral hepatitis, alcoholic liver disease, and non-alcoholic fatty liver disease [7]. The liver is the major site of clearance and metabolism of biologicallyactive TNF- $\alpha$ [8].

Alcohol consumption is a major risk factor for liver disease. Alcoholic liver disease (ALD) is associated with increased production of pro-inflammatory cytokines. Chronic alcohol drinking triggers the sympathetic hyperactivity-activated hepatic stellate cell feedback loop that activates the HSCs, resulting in HSC-derived TNF- $\alpha$ overproduction [9]. TNF- $\alpha$ is the central mediator of apoptotic and necrotic liver damage. TNF- $\alpha$ may be harmful, leading to initiation of fibrosis, cirrhosis, and, eventually HCC [10]. TNF-a produces oxygenderived free radicals and other reactive oxygen species. These compounds are important mediators of hepatic fibrogenesis in liver injury $[11,12]$. TNF- $a$ may induce the production of other fibrogenic factors, such as tumour growth factor $\beta$ and interleukins (IL- 1,IL-6, IL-8, and IL-10) [13].

Daniluk et al. have shown that a significant derangement of the balance between pro-inflammatory and antiinflammatory signals was characteristic of compensated and especially of decompensated alcoholic cirrhosis. Patients with compensated alcoholic cirrhosis showed increased TNF- $\alpha$, IL-8, and IL-2 levels comparable to those of controls. In contrast, patients with decompensated cirrhosis showed significantly higher levels of TNF- $\alpha$, IL-2, IL-6 and IL-8, than controls [14]. In contrast to the current study, Daniluk et al. did not analyzed the results in subgroups of various stages of liver cirrhosis according to the Pugh-Child criteria.

In the presented study, serum TNF- $\alpha$ concentrations were significantly higher in cirrhotic patients than in controls. The concentration of TNF- $\alpha$ in the group if patients with stage $\mathrm{C}$ and $\mathrm{B}$ liver cirrhosis were significantly higher than those in control group. Similar results were reported by Shiraki et al., who additionally demonstrated that high a activity of the TNFa system mediates the aberrant energy metabolism in patients with liver cirrhosis [15].

Apoptosis is defined as programmed cell death, and it plays a role in the development and homeostasis of multicellular organisms 16]. CK-18 is a normal component of the hepatocyte cytoskeleton. Apoptosis of hepatocytes is associated with the release of caspase-cleaved CK-18 fragments in the bloodstream [17].

Assessment of CK-18 cell death biomarkers allows for early detection of liver damage in acute and chronic liver diseases. CK-18 fragments in the circulation can be detected via the ELISA method using M30 and M65 antibodies [18]. Kar et al. have determined the role of serum apoptosis markers M30 and M65 as the prognostic markers in patients with acute liver failure. The levels of total CK-18 (M65) were significantly higher in non-survivors compared to survivors in the course of acute liver failure [19]. In the current study, the extent of hepatocyte death (both through apoptosis and necrosis) according to the Pugh-Child criteria in patients with alcoholic liver cirrhosis, was assessed using M65 concentrations. 
The concentration of CK-18 (biomarker m65) was statistically higher in patients with alcoholic liver cirrhosis compared to the control group. There were no statistically significant differences in CK-18 according to the stage of alcoholic liver cirrhosis. Moreover, no correlations were observed the level of TNF- $\alpha$ and of CK-18 in the group of patients with alcoholic liver cirrhosis, which is likely to evidence that some other than TNF- $\alpha$ metabolic pathways affecting the death of hepatocytes can be involved in the group of patients with alcoholic liver cirrhosis. Further studies are required to determine them. Another possibility is that the concentration of TNF- $\alpha$ does not reflect accurately the paracrine action of this cytokine directly in the liver.

Gonzales-Quintela et al. have confirmed that serum CK-18 levels are increased in drinkers with alcoholic liver disease 20]. CK-18 is suggested to be a marker of the histological degree of inflammation and liver damage clearly exceeding transaminases [21].

Joka et al. analyzed sera from patients with non-alcoholic chronic liver diseases and different fibrosis stages for caspase-cleaved fragments and total CK-18 levels. They found a significant correlation between CK-18 levels and both liver stiffness and histological fibrosis. Compared with the apoptosis biomarker M30, the M65 assay had a better diagnostic performance and even differentiated between lower fibrosis stages, as well as between healthy individuals and patients with simple steatosis [22].

Lavallard et al. have demonstrated that circulating levels of total and the caspase-generated fragments of CK-18 predict with good accuracy severe fibrosis in heavy alcohol drinkers. These markers also correlate with hepatocyte ballooning, the presence of Mallory-Denk bodies and hepatic and TGF $\beta$ expression [23]. As opposed to the presented findings, Lavallard et al. found a significant correlation of moderate strength between the expression of TNF-a gene in liver bioptates and the concentration of CK-18. It should be emphasized that their study was performed in a group of 24 patients with alcoholic liver disease, and not in a selected subgroup of patients with alcoholic liver cirrhosis, as was the case in the current study.

Furthermore, there are studies regarding CK-18 in nonalcoholic live diseases. Więckowska et al. have shown that levels of CK-18 fragments might allow the discrimination between NASH and NAFL patients. Therefore, CK-18 may be useful for the diagnosis of NASH [24].

The study of Ding et al. has suggested that the circulating M65 may serve as a relatively independent prognostic parameter for liver failure in type B hepatitis patients [25].

\section{CONCLUSIONS}

The levels of TNF- $\alpha$ and total CK-18 are higher in patients with alcoholic liver cirrhosis compared to healthy individuals. The concentration of TNF- $\alpha$ increases with decompensated cirrhoiss (stage B and C). Otherwise, the level of CK-18 was not correlated with the stage of liver cirrhosis. Moreover, there was no significant correlation between CK-18 and TNF- $\alpha$. Further studies are needed regarding the prognostic value of CK-18 in this group of patients.

\section{Acknowledgement}

This study was supported by grants from the Medical University in Lublin, Poland (Grant No. DS 507/2013-2015).

\section{Conflict of Interest Statement}

The authors state that there are no conflicts of interest regarding the publication of this article

\section{REFERENCES}

1.Zatoński W, Sułkowska U, Mańczuk M, et al. Liver Cirrhosis Mortality in Europe, with Special Attention to Central and Eastern Europe. Eur Addict Res. 2010;16: 193-201.

2. Schuppan D, Afdhal N. Liver Cirrhosis. Lancet 2008; 371: 838-51.

3. Olaso E, Friedman S. Molecular regulation of hepatic fibrogenesis. J Hepatol. 1998; 29: 836-47.

4. Rath P, Aggarwal B. TNF-induced signaling in apoptosis. J Clin Immunol. 1999; 19: 350-64.

5. Ustaalioglu B, Bilici A, Ercan S, et al. The prognostic importance of changing serum M30 and M65 values after chemotherapy in patients with advanced-stage non-small-cell lung cancer. Med Oncol. 2013; 30: 551.

6. Hagg M, Biven K, Ueno T, et al. A novel high-through-put assay for screening of pro-apoptotic drugs. Invest New Drugs. 2002; 20: 253-9.

7. Szabo G, Mandrekar P, Dolganiuc A. Innate immune response and hepatic inflammation. Semin Liver Dis. 2007; 27: 339-50.

8. Bemelmans M, van Tits L, Buurman W. Tumour necrosis factor: function, release and clearance. Crit Rev Immunol. 1996; 16: 1-11.

9. Liu J. Ethanol and liver: recent insights into the mechanisms of ethanolinduced fatty liver. World J Gastroenterol. 2014; 20: 14672-85.

10. Jen-Eing T, Jung-Fa C, Lee-Yea H, et al. Tumour necrosis factor- $\alpha 308.2$ polymorphism is associated with advanced hepatic fibrosis and higher risk for hepatocellular carcinoma. Neoplasia 2007; 9: 987-92.

11. Giannelli G, Antonaci S. Immunological and molecular aspects of liver fibrosis in chronic hepatitis $\mathrm{C}$ virus infection. Histol Histopathol. 2005; 20: 939-44.

12. Bataller R, Brenner D. Liver fibrosis. J Clin Invest. 2005; 115: 209-18.

13. Balkwill F. Cancer and the chemokine network. Nat Rev Cancer. 2004; 4: $540-50$.

14. Daniluk J, Szuster-Ciesielska A, Drabko J, et al. Serum cytokine levels in alcohol-related liver cirrhosis. Alcohol. 2001; 23: 29-34.

15. Shiraki M, Terakura Y, Iwasa J, et al. Elevated serum tumour necrosis factor-a and soluble tumour necrosis factor receptors correlate with aberrant energy metabolism in liver cirrhosis. Nutrition. 2010; 26: 269-75.

16. Niknejad H, Khayat-Khoei M, Peirovi H, et al. Human amniotic epithelial cells induce apoptosis of cancer cells: a new anti-tumour therapeutic strategy. Cytotherapy 2014; 16: 33-40.

17. Canbay A, FeldsteinA, Kronenberger B, et al. Cytokeratin 18 as marker for non-invasive diagnosis and prognosis of acute and chronic liver diseases. Z Gastroenterol. 2014; 52: 290-5.

18. Yildiz I, Tas F, Kilic L, et al. A high serum level of M65 is associated with tumour aggressiveness and an unfavourable prognosis for epithelial ovarian cancer. Cancer Chemother Pharmacol. 2013; 72: 437-44.

19. Kar P, Malik P. Prognostic Value of Serum Apoptosis Markers CK 18 [M30 and M65] in Acute Liver Failure. J Clin Exp Hepatol. 2014; 4(suppl. 2): S1-2.

20. Gonzalez-Quintela A, García J, Campos J, et al. Serum cytokeratins in alcoholic liver disease: contrasting levels of cytokeratin-18 and cytokeratin-19. Alcohol. 2006; 38: 45-9.

21. Mueller S, Seitz H, Rausch V. () Non-invasive diagnosis of alcoholic liver disease. World J Gastroenterol. 2014; 20: 14626-41.

22. Joka D, Wahl K, Moeller S, et al. Prospective biopsy-controlled evaluation of cell death biomarkers for prediction of liver fibrosis and nonalcoholic steatohepatitis. Hepatol. 2012; 55: 455-64.

23. Lavallard V, Bonnafous S, Patouraux S, et al. Serum Markers of Hepatocyte Death and Apoptosis Are Non Invasive Biomarkers of Severe Fibrosis in Patients with Alcoholic Liver Disease. PLoS ONE 2011; 6: e17599. doi:10.1371/journal.pone.0017599.

24. Wieckowska A, Zein N, Yerian L, et al. In vivo assessment of liver cell apoptosis as a novel biomarker of disease severity in nonalcoholic fatty liver disease. Hepatol. 2006; 44: 27-33.

25. Ding G, Li Z, Zhang L, et al. Circulating full-length cytokeratin 18 for predicting prognosis of hepatitis B virus-related acute-on-chronic liver failure patients with lamivudine treatment. Hepatol Res. 2016; 46(4): 329-334. doi: 10.1111/hepr.12549. 